\title{
Electron transport through multilevel quantum dot
}

\author{
Santanu K. Maiti ${ }^{1,2, *}$ \\ ${ }^{1}$ Theoretical Condensed Matter Physics Division, Saha Institute of Nuclear Physics, \\ 1/AF, Bidhannagar, Kolkata-700 064, India \\ ${ }^{2}$ Department of Physics, Narasinha Dutt College, 129, Belilious Road, Howrah-711 101, India
}

\begin{abstract}
Quantum transport properties through some multilevel quantum dots sandwiched between two metallic contacts are investigated by the use of Green's function technique. Here we do parametric calculations, based on the tight-binding model, to study the transport properties through such bridge systems. The electron transport properties are significantly influenced by (a) number of quantized energy levels in the dots, (b) dot-to-electrode coupling strength, (c) location of the equilibrium Fermi energy $E_{F}$ and (d) surface disorder. In the limit of weak-coupling, the conductance $(g)$ shows sharp resonant peaks associated with the quantized energy levels in the dots, while, they get substantial broadening in the strong-coupling limit. The behavior of the electron transfer through these systems becomes much more clearly visible from our study of current-voltage $(I-V)$ characteristics. In this context we also describe the noise power of current fluctuations $(S)$ and determine the Fano factor $(F)$ which provides an important information about the electron correlation among the charge carriers. Finally, we explore a novel transport phenomenon by studying the surface disorder effect in which the current amplitude increases with the increase of the surface disorder strength in the strong disorder regime, while, the amplitude decreases in the limit of weak disorder. Such an anomalous behavior is completely opposite to that of bulk disordered system where the current amplitude always decreases with the disorder strength. It is also observed that the current amplitude strongly depends on the system size which reveals the finite quantum size effect.
\end{abstract}

PACS No.: 73.23.-b; 73.63.Rt; 85.65.+h

Keywords: Green's function; Quantum dot; Conductance; $I$ - $V$ characteristic; Fano factor; Surface disorder; Bulk disorder.

*Corresponding Author: Santanu K. Maiti

Electronic mail: santanu.maiti@saha.ac.in 


\section{Introduction}

More advances in nano-science and technology have made feasible to growth nanometer sized systems, like quantum wires [1, quantum dots [2, 3, 4] and molecular wires [5. Electronic transport properties in such systems have attracted much more attention since these are the fundamental building blocks for future generation of electronic devices. There has also been an growing interest in deriving analytical results for electron transport in quantum dots, molecular wires and single molecule systems. The electron transport properties through molecular bridge systems were first studied theoretically in 1974 by Aviram et al. [6]. Since then several numerous experiments [7, 8, 9, 10, 11. have been performed through molecules placed between two electrodes with few nanometer separation. Full quantum mechanical treatment is required to characterize the transport in such systems. The transport properties are characterized by several significant factors like as the quantization of energy levels, quantum interference of electron waves 12, 13, 14, 15, 16, 17 associated with the geometry of the bridging system adopts within the junction and other different parameters of the Hamiltonian that are needed to describe the complete system. The knowledge of current fluctuations (of thermal or quantum origin) also provides several key ideas for fabrication of efficient molecular devices. In a review work Blanter et al. 18 have described clearly and elaborately how the lowest possible noise power of the current fluctuations can be determined in a two-terminal conductor. The steady state current fluctuations so-called shot noise is a consequence of the quantization of charge and it can be used to obtain information on a system which is not directly available through conductance measurements. The noise power of the current fluctuations gives an additional important information about the electron correlation by calculating the Fano factor $(F)$ which directly informs us whether the magnitude of the shot noise achieves the Poisson limit $(F=1)$ or the sub-Poisson $(F<1)$ limit.

Quantum dots are man-made "droplets" of charge that can contain anything from a single electron to a collection of several thousand. Their typical dimensions range from nanometers to a few microns, and their size, shape and interactions can be precisely controlled through the use of advanced nanofabrication technology. A quantum dot can also be assumed as an artificial molecule with few number of atoms and several phenomena can be studied by allowing single electron to tunnel into and out of the dot, since the quantum dot reveals quantized energy levels, and, here we concentrate our study on the electron transport through such a dot.

There exist several ab initio methods for the calculation of conductance [19, 20, 21, 22, 23, 24. through a molecular bridge system. At the same time the tight-binding model has been extensively studied in the literature and it has also been extended to DFT transport calculations 25. The study of static density functional theory (DFT) 26] within the local-density approximation (LDA) to investigate the electron transport through nanoscale conductors, like atomic-scale point contacts, has met with great success. But when this similar theory applies to molecular junctions, theoretical conductances achieve larger values compared to the experimental predictions and these quantitative discrepancies need extensive study in this particular field. In a recent work, Sai et al. 27] have predicted a correction to the conductance using the time-dependent current-density functional theory since the dynamical effects give significant contribution in the electron transport, and illustrated some important results with specific examples. Similar dynamical effects have also been reported in some other recent papers 28, 29, where authors have abandoned the infinite reservoirs, as originally introduced by Landauer, and considered two large but finite oppositely charged electrodes connected by a nanojunction. In this article we reproduce an analytic approach based on the tight-binding model to characterize the electron transport properties through some quantum dots placed between two macroscopic contacts. We utilize a simple parametric approach [30, 32, 31, 33, 34, 35, 36 for these calculations. The model calculations are motivated by the fact that the $a b$ initio theories are computationally much more expensive, while, the model calculations by using the tight-binding formulation are computationally very cheap and also provide a worth insight to the problem. In this context we also explore a novel feature of electron transport by considering the effect of surface disorder on the dot. Advanced nanoscience and technology can easily fabricate a mesoscopic device in which charge carriers are scattered mainly by the surface boundaries and not by the impurities located in the core region [37, 38, 39. The idea of such a system named as shell-doped nanowires has been given in recent works by Zhong et al. 40, 41 where the carrier mobility can be controlled nicely. The shell-doping 
confines the dopant atoms spatially within a few atomic layers in the shell region of nanowire. This is completely opposite to that of the conventional doping where the dopant atoms are distributed uniformly inside the nanowire. Such a system provides a novel feature of electron transport in which the current amplitude increases with the increase of the surface disorder strength in the limit of strong disorder, while, the current amplitude decreases in the weak disorder limit. To emphasize such an interesting phenomenon, here we describe the electron transport through a quantum dot in which the impurities are located only in its surface boundary. It is also noticed that the electron transport through the dot is significantly influenced by the number of quantized energy levels of the dot which manifests the finite quantum size effects.

Our scheme of study is as follows. Section 2 describes the theoretical formalism of our study. In Section 3 we focus and explain our significant results and see that the electron transport properties are significantly influenced by (a) number of quantized energy levels in the dots, (b) dot-to-electrode coupling strength, (c) location of $E_{F}$ and (d) surface impurity. Finally, we draw our conclusions in Section 4.

\section{Description of model and formalism}

Here we describe very briefly the methodology for the calculation of the transmission probability $(T)$, conductance $(g)$, current $(I)$ and the noise power of its fluctuations $(S)$ through a quantum dot attached to two metallic electrodes (schematically illustrated as in Fig. 1) by using the Green's function technique.

At sufficient low temperature and small applied voltage the conductance $g$ of the dot is expressed through the Landauer conductance formula 42,

$$
g=\frac{2 e^{2}}{h} T
$$

where the transmission probability $T$ is written in this form 42,

$$
T=\operatorname{Tr}\left[\Gamma_{S} G_{d o t}^{r} \Gamma_{D} G_{d o t}^{a}\right]
$$

In this expression $G_{d o t}^{r}$ and $G_{d o t}^{a}$ are the retarded and advanced Green's functions of the dot and $\Gamma_{S}$ and $\Gamma_{D}$ describe its (dot) coupling to the source and drain, respectively. The Green's function of the multilevel quantum dot is expressed as,

$$
G_{\mathrm{dot}}=\left(E-H_{\mathrm{dot}}-\Sigma_{S}-\Sigma_{D}\right)^{-1}
$$

where $E$ is the energy of the injecting electron and $H_{d o t}$ is the Hamiltonian of the dot (here the quantum dot is assumed as an artificial molecule with

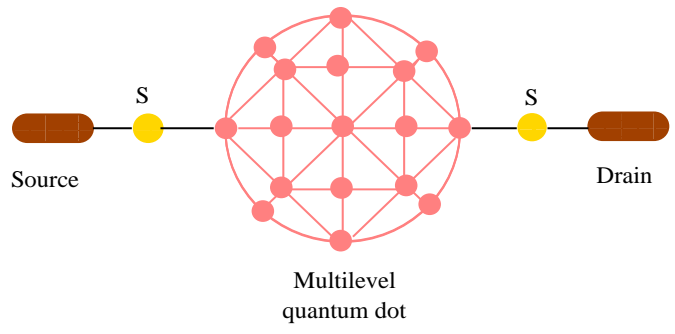

Figure 1: (Color online). Schematic representation of a multilevel quantum dot attached to two metallic electrodes (source and drain) through lattice sites $S$ and $S$ where the filled red circles correspond to the atomic sites in the dot.

several number of atoms) which can be written in the tight-binding model within the non-interacting picture like,

$$
H_{\mathrm{dot}}=\sum_{i} \epsilon_{i} c_{i}^{\dagger} c_{i}+\sum_{<i j>} t\left(c_{i}^{\dagger} c_{j}+c_{j}^{\dagger} c_{i}\right)
$$

where $\epsilon_{i}$ 's are the site energies and $t$ is the hopping strength between two nearest-neighbor atomic sites in the dot. To introduce the impurities in the dot we choose the site energies $\left(\epsilon_{i}\right.$ 's) in the form of incommensurate potentials through the expression $\epsilon_{i}=\sum_{i} W \cos (i \lambda \pi)$ where $\lambda$ is an irrational number and $W$ is the strength of the disorder. As a typical example we take the value of $\lambda$ as the golden mean $(1+\sqrt{5}) / 2$. Setting $\lambda=0$ we get back the pure system with identical site potential $W$. In Eq.(3), $\Sigma_{S}$ and $\Sigma_{D}$ correspond to the self-energies due to coupling of the dot to the two electrodes. These two semi-infinite onedimensional metallic electrodes are described also by the similar kind of tight-binding Hamiltonian as given in Eq.(4), where we take the site energy and the nearest-neighbor hopping strength by the parameters $\epsilon_{i}^{\prime}$ and $v$, respectively. All the information about the dot-to-electrode coupling are included into these two self-energies as stated above and are described through the use of Newns-Anderson chemisorption theory [30, 31]. The detailed description of this theory is available in these two references. By utilizing the Newns-Anderson type model 
we can express the conductance in terms of the effective dot properties multiplied by the effective state densities involving the coupling. This allows us to study directly the conductance as a function of the properties of the electronic structure of the dot between the electrodes.

The current passing through the dot can be considered as a single electron scattering process between the two reservoirs of charge carriers. The current-voltage $(I-V)$ relationship can be computed from the expression 42 ,

$$
I(V)=\frac{e}{\pi \hbar} \int_{-\infty}^{\infty}\left(f_{S}-f_{D}\right) T(E) d E
$$

where $f_{S(D)}=f\left(E-\mu_{S(D)}\right)$ gives the Fermi distribution function with the electrochemical potentials $\mu_{S(D)}=E_{F} \pm \mathrm{eV} / 2$. For the sake of simplicity, here we assume that the entire voltage is dropped across the dot-electrode interfaces and this assumption does not greatly affect the qualitative aspects of the $I-V$ characteristics. Such an assumption is based on the fact that the electric field inside the dot, especially for the dots with smaller number of atomic sites, seems to have a minimal effect on the conductance-voltage characteristics. On the other hand, for the dots with very large number of atomic sites and high bias voltage, the electric field inside the dot may play a more significant role depending on the internal structure of the dot 43 , yet the effect is much small.

The noise power of the current fluctuations is calculated through the following relation [18,

$$
\begin{aligned}
S= & \frac{2 e^{2}}{\pi \hbar} \int_{-\infty}^{\infty}\left[T(E)\left\{f_{S}\left(1-f_{S}\right)+f_{D}\left(1-f_{D}\right)\right\}\right. \\
& \left.+T(E)\{1-T(E)\}\left(f_{S}-f_{D}\right)^{2}\right] d E
\end{aligned}
$$

where the first two terms in this equation correspond to the equilibrium noise contribution and the last term gives the non-equilibrium or shot noise contribution to the power spectrum. By calculating the noise power of the current fluctuations we can evaluate the Fano factor $F$, which is essential to predict whether the shot noise lies in the Poisson or the sub-Poisson regime, through the relation [18],

$$
F=\frac{S}{2 e I}
$$

The shot noise achieves the Poisson limit when $F=1$ and in this case no electron correlation exists between the charge carriers. On the other hand, for $F<1$ the shot noise reaches the sub-Poisson limit and it provides the information about the electron correlation among the charge carriers.

In this article we perform all the calculations at absolute zero temperature, but the qualitative behavior of all the results are invariant up to some finite (low) temperature. The reason for such an assumption is that the broadening of the energy levels of the dot due to its coupling to the electrodes is much larger than that of the thermal broadening. For simplicity, we take the unit $c=e=h=1$ in our present investigation.

\section{Results and discussion}

This section demonstrates the transport properties of some multilevel quantum dots in the coherent
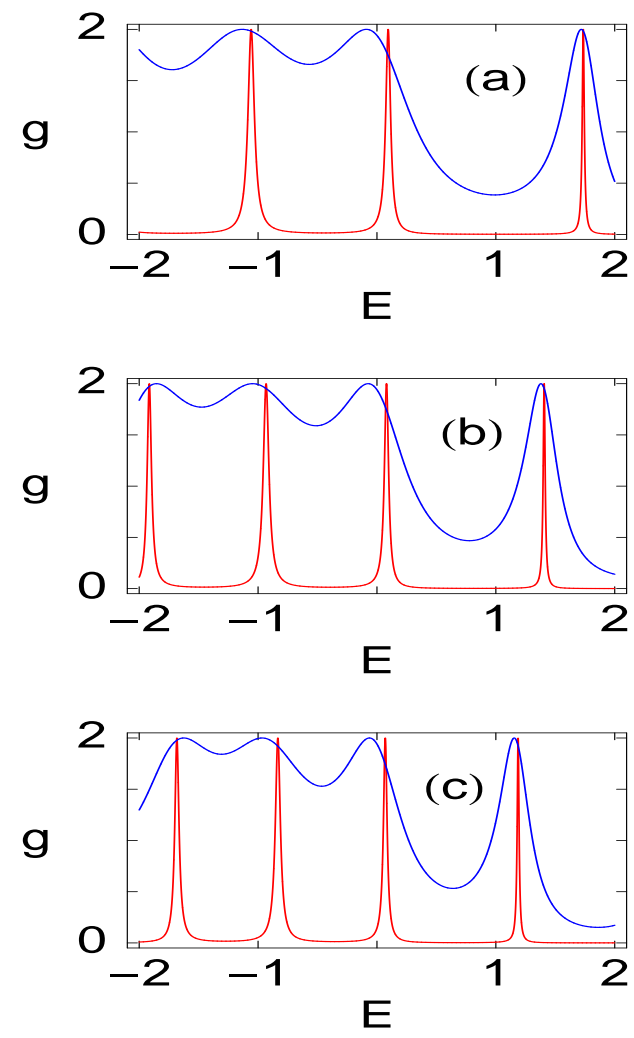

Figure 2: (Color online). $g$ - $E$ characteristics for the multilevel quantum dots, where (a), (b) and (c) are respectively for the dots with 14, 18 and 22 atomic sites. The red and blue curves correspond to the weak- and strong-coupling cases, respectively.

transport regime. In the bridge system, the dot is 
attached to two metallic electrodes through the lattice sites $S$ and $S$, as schematically illustrated in Fig. 1. In actual experimental set up, these two electrodes made from gold are used and the dot attached to them via thiol groups in the chemisorption technique and in making such contact with these electrodes, the hydrogen $(\mathrm{H})$ atoms of the thiol groups remove and the sulfur $(\mathrm{S})$ atoms reside. In our tight-binding formulation, the quantum dot is coupled to the electrodes through the lattice sites $S$ and $S$ by using the parameters $\tau_{S}$ and $\tau_{D}$, where they (coupling parameters) correspond to the coupling strengths to the source and drain, respectively. Here we concentrate our results on clarifying the following points: the dependence of the conductance, current and the noise power of its fluctuations on (I) the number of energy levels in the dot and (II) dot-to-electrode coupling strength and also discuss the dependence of the current and its fluctuations on the location of the equilibrium Fermi energy $E_{F}$. Finally, attention is drawn on the study of the surface disorder effect in such electron transport. Throughout this article we will discuss all the essential features of the electron transport for the two distinct regimes. One is the so-called weak-coupling regime denoted by the condition $\tau_{\{S, D\}}<<t$ and the other one is the so-called strong-coupling regime where $\tau_{\{S, D\}} \sim t$. The values of such parameters for these two distinct regimes are chosen as $\tau_{S}=\tau_{D}=0.5, t=2.5$ (weak-coupling) and $\tau_{S}=\tau_{D}=2, t=2.5$ (strongcoupling).

The characteristic behavior of the conductance $g$ as a function of the injecting electron energy $E$ for the multilevel quantum dots are shown in Fig. 2. where (a), (b) and (c) correspond to the dots with 14, 18 and 22 atomic sites, respectively. The red lines represent the results in the limit of weak-coupling, while, the blue lines denote the results for the strong-coupling limit. In the weakcoupling limit, the conductance shows very sharp resonant peaks (red curves in Fig. 2) for some particular energy values, while, for all other energies it almost vanishes. At these resonances the conductance $g$ achieves the value 2 , and accordingly, the transmission probability $T$ goes to unity, since from the Landauer conductance formula we get $g=2 T$ (see Eq.(1) with $e=h=1$ in our present description). These resonant peaks are associated with the energy eigenvalues of the corresponding dot and therefore more resonant peaks appear with the increase of the quantized energy levels in the dot. Thus it can be emphasized that the conduc- tance spectrum manifests itself the energy eigenvalues of the dot. With the increase of the dotto-electrode coupling strength, the widths of these resonances get enhanced substantially, as illustrated by the blue curves in Fig. 2. This is due to the substantial broadening of the quantized energy levels in the limit of strong-coupling. The contribution for such broadening of the energy levels comes from the imaginary parts of the two self energies $\Sigma_{S}$ and $\Sigma_{D}$, respectively 42 . Thus for the strong-coupling limit, the electron conducts through the dots for the wide range of energies, while, a fine tuning in the energy scale is necessary to get the electron conduction through these systems in the limit of weakcoupling. Therefore, it can be predicted that the dot-to-electrode coupling strength has a significant role in the determination of the electron conduction through the bridge systems. This feature provides a key information in the study of molecular transport phenomena.

The behavior of the electron transfer through such systems can be described much more clearly by studying the current-voltage $(I-V)$ characteristics. In the forthcoming parts we will concentrate our study on the current and the noise power of its fluctuations $(S)$ as a function of the applied bias voltage $(V)$ for these quantum dots. Both the current and the noise power of its fluctuations are determined from the integration method of the transmission function $(T)$ as described in Eq.(5) and in Eq.(6), where the transmission function varies exactly similar to that of the conductance spectra as illustrated in Fig. 2 differ only in magnitude by the factor 2 since the relation $g=2 T$ holds from the Landauer conductance formula (Eq.(1)). In Fig. 3, we display the variation of the current and the noise power of its fluctuations as a function of the applied bias voltage for the multilevel quantum dots in the limit of weak-coupling, where the first, second and third columns are respectively for the dots with 14, 18 and 22 atomic sites. The red line corresponds to the current and the blue line represents the noise power of its fluctuations. In order to emphasize the effect of the location of the Fermi energy $E_{F}$ on such transport here we plot the results considering three different values of $E_{F}$, where the first, second and third rows correspond to the results for the dots with $E_{F}=0,-0.5$ and -1 , respectively. The current shows staircase-like structure with sharp steps as a function of the applied bias voltage. This is due to the existence of the sharp resonant peaks in the conductance spectra (see the red curves in Fig. 2) in this limit of weak-coupling, since we com- 

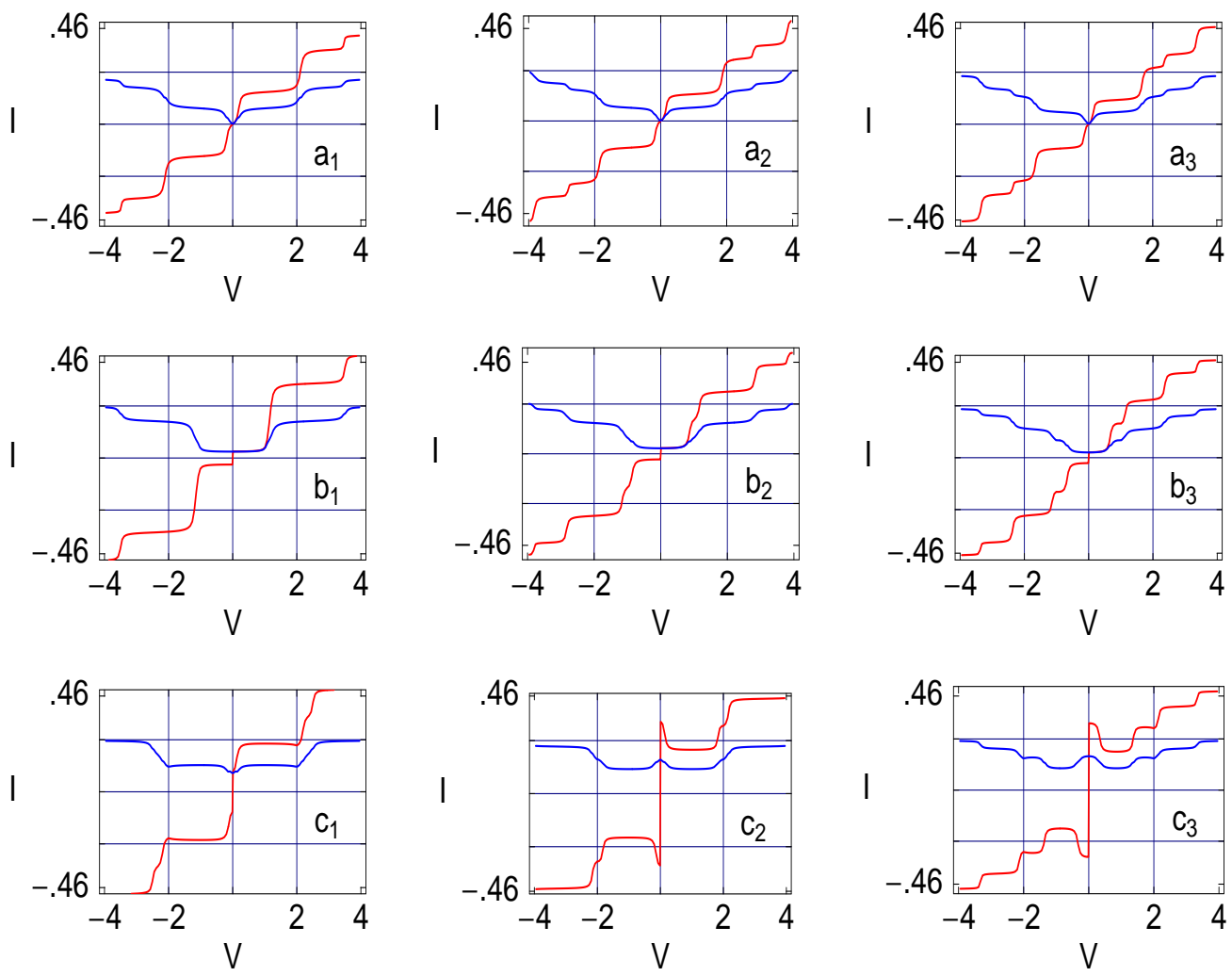

Figure 3: (Color online). Current $I$ (red curve) and the noise power of its fluctuations $S$ (blue curve) as a function of the applied bias voltage $V$ for the multilevel dots in the limit of weak-coupling, where the 1st, 2nd and 3rd columns are respectively for the dots with 14, 18 and 22 atomic sites. The 1st, 2nd and 3 rd rows correspond to the results for the dots with the Fermi energy $E_{F}=0,-0.5$ and -1 , respectively.

pute the current from the integration procedure of the transmission function $T$. The electrochemical potentials on the electrodes are shifted gradually with the increase of the applied bias voltage and eventually cross one of the quantized energy levels in the dot. Accordingly, a current channel is opened up and the current-voltage characteristic curve produces a jump. With the increase of the dot size i.e., number of quantized energy levels, current shows more steps (as expected) which is clearly visible from this Fig. 3 . For all these bridges we observe that the current amplitudes are too small and they are comparable with each other (see the red lines in Fig. (3). Now we discuss the effect of the location of the Fermi energy $E_{F}$ in these quantum dots. The effect is quite interesting. We see that for $E_{F}=0$, the current amplitude for all these three bridges across $V=0$ is almost zero, while, the amplitude gradually increases across this voltage $(V=0)$ as we change the Fermi energy $E_{F}$ to the values -0.5 and -1 , respectively. For $E_{F}=-1$, we get a very large current compared to the other two values of $E_{F}$. For a particular coupling strength this current amplitude depends on both the location of the Fermi energy and the number of the quantized energy levels in the dots. Thus it can be predicted that, by tuning the Fermi energy we can get the on/off state of the bridge system across $V=0$. This is an important finding in the study of molecular transport. Now in the determination of the noise power of the current fluctuations we get several interesting results depending on the values of $E_{F}$ and the number of quantized energy levels. Both for the choices of $E_{F}=0$ and -1 , the shot noise (blue curves in the 1st and the 3rd rows of Fig. (3) lies in the sub-Poisson regime $(F<1)$ momentarily as we switch on the bias voltage. Accordingly, for such cases the electrons are always correlated with each other. Here the correlation of the electrons means one electron feels the existence of the other in the 

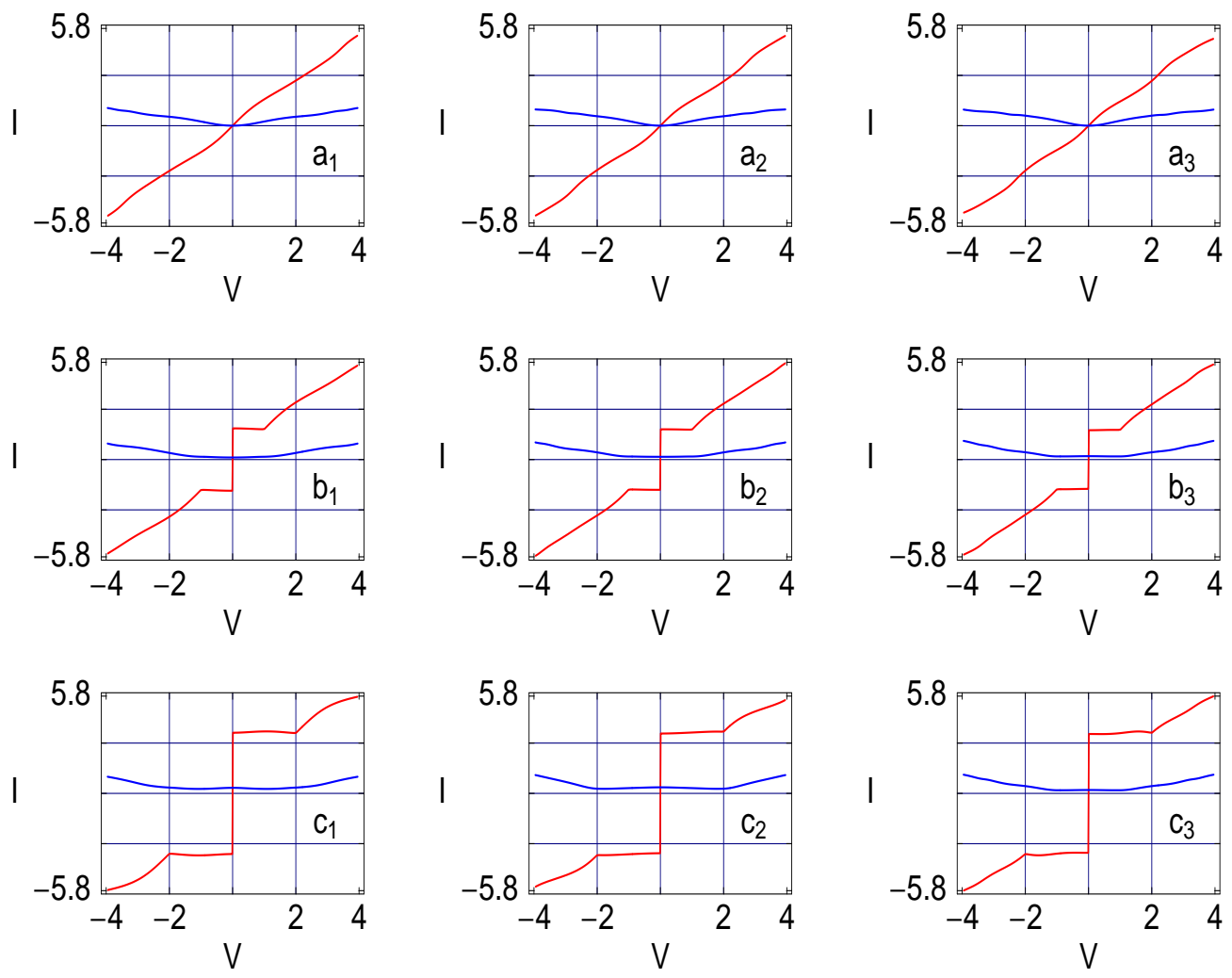

Figure 4: (Color online). Current $I$ (red curve) and the noise power of its fluctuations $S$ (blue curve) as a function of the applied bias voltage $V$ for the multilevel dots in the limit of strong-coupling, where the 1st, 2nd and 3rd columns are respectively for the dots with 14, 18 and 22 atomic sites. The 1st, 2nd and 3 rd rows correspond to the results for the dots with the Fermi energy $E_{F}=0,-0.5$ and -1 , respectively.

sense of Pauli exclusion principle, since no other electron-electron interaction is taken into account in our present description. On the other hand for the case where we set $E_{F}=-0.5$, the shot noise (blue curves in the 2nd row of Fig. 3) makes a transition from the Poisson limit $(F=1)$ to the sub-Poisson limit $(F<1)$ as long as we cross the first step in the current-voltage characteristics. This indicates that the electrons are correlated after the tunneling process has occurred. For such a particular case $\left(E_{F}=-0.5\right)$, it is also observed that the threshold bias voltage $\left(V_{t h}\right)$ where the shot noise makes a transition from the Poisson to the sub-Poisson limit gradually decreases with the increase of the quantized energy levels in the quantum dots. Another important observation is that, for all these three bridge systems the noise power of the current fluctuations remains in the same level independent of the number of the quantized energy levels i.e., the number of atomic sites in the dots.
The characteristic features of the current and the noise power of its fluctuations are also very interesting for these bridges in the limit of strong-coupling. The results are shown in Fig. 4, where the figures in the different rows and columns correspond to the same meaning as presented in Fig. 3. The red and blue curves also represent the identical meaning as in Fig. 3. From the results plotted in Fig. 4 it is observed that, both the current and noise power vary quite continuously as a function of the applied bias voltage $V$. Such kind of behavior appears due to the broadening of the resonant peaks (blue curves in Fig. 2) in the limit of strong-coupling, since the current and the noise power are determined from the integration procedure of the transmission function $T$. One key result is that, the current amplitudes get enhanced quite significantly compared to the current amplitudes obtained in the limit of weak-coupling (see the red curves in Fig. 3). This can be understood by noting the areas under the 
curves in the conductance spectra for the two limiting cases as plotted in Fig. 2. Thus by tuning the dot-to-electrode coupling strength one can achieve greater current across the bridge system which also provides an interesting phenomenon in fabrication of molecular devices. Lastly, in the study of the noise power of the current fluctuations we find that, for all the choices of the Fermi energy $E_{F}$ there is no such possibility of transition from the Poisson limit to the sub-Poisson limit, since the shot noise already achieves the sub-Poisson limit (blue curves of Fig. 4) momentarily as we apply the bias voltage. Therefore, for all such values of $E_{F}$ the electron correlation is more significant. Thus we can emphasize that, both the dot-to-electrode coupling strength and the location of the Fermi energy are the key factors that control the electron transport through a bridge system.

Now we concentrate our study on the correlation effect between the surface disorder and bulk disorder on the electron transport through the multilevel quantum dots. The schematic representation of a surface disordered multilevel quantum dot attached to two metallic electrodes is shown in Fig. 5 The disorder in the surface is represented by the filled black circles of different sizes which correspond to the different atomic sites with variable site energies, while, the inner core perfect region is described by the filled red circles with identical site energies. In order to introduce the impurities, we choose the site energies $\left(\epsilon_{i}\right.$ 's) from the incommensurate potential distribution function as stated earlier in Section 2. For the surface disordered system, the impurities are given only in the atomic sites located in the surface, while, for the bulk disordered case the impurities are introduced in all the atomic sites. Here we use the parameters $N_{s}$ and $N_{c}$ to denote the total number of atomic sites in the surface boundary and in the core region of the dot, respectively. For the sake of simplicity, we set the equilibrium Fermi energy $E_{F}=0$ in this particular study. Figure 6 shows the variation of the current amplitudes $\left(I_{0}\right)$, in the strong-coupling limit, as a function of the impurity strength $(W)$ for the multilevel quantum dots with $N_{s}=20$ and $N_{c}=15$. The current amplitudes are computed at the typical bias voltage $V=1.5$, where the red and blue curves correspond to the results for the surface and bulk disordered cases, respectively. Since we introduce only the diagonal disorder by considering the site energies from the known incommensurate potential distribution function $\left(\epsilon_{i}=\sum_{i} W \cos (i \lambda \pi)\right.$ with $\left.\lambda=(1+\sqrt{5}) / 2\right)$ we do not take any disorder averaging during our calculation. The idea of considering such kind of potential distribution function rather than any random distribution function is to avoid the disorder averaging over large number of possible disordered configurations since it takes too much time to evaluate the results. Now instead of considering such an unconventional incommensurate potential func-

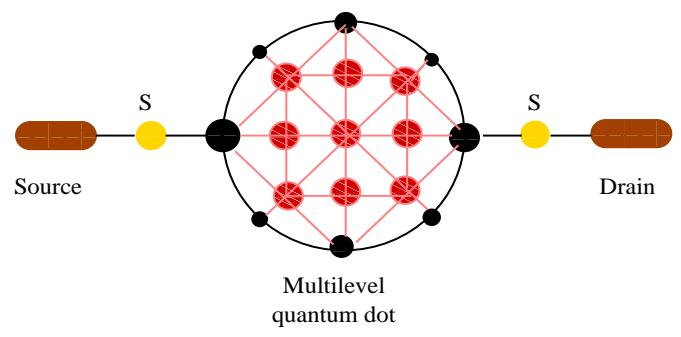

Figure 5: (Color online). Schematic view of a surface disordered multilevel quantum dot attached to two metallic electrodes (source and drain) through lattice sites $S$ and $S$. Disorder in the surface of the dot is represented by the filled black circles of different sizes which correspond to the different lattice sites with variable site energies.

tion, we can also take any random distribution function and in that case we have to take the average over a large number of random disordered configurations to achieve much more accurate result. Both these two different treatments of the disorder in the model provide quite similar in nature for the variation of the current amplitude and due to this fact we choose the unconventional treatment of the disorder, instead of the other one, to understand the results through limited numerical resources. From the results it is observed that, in the bulk disordered case the current amplitude gradually decreases with the increase of the impurity strength and for the strong enough impurity it almost drops to zero. This behavior can be well understood from the theory of Anderson localization where the states become more localized with the increase of the impurity strength. The significant feature appears when the impurities are given only in the surface boundary of the dot. The current amplitude initially decreases with the strength of the impurity, while, beyond some critical value of the impurity strength $W=W_{c}$ (say) the amplitude increases. Such an anomalous behavior is completely opposite to that of the bulk disordered case and it can be explained as follows. In the ordered-disordered separated quantum dot, a gradual separation of the energy spectra of the disordered surface and the 
perfect inner core regions takes place with the increase of the disorder strength $W$. Accordingly, the influence of random scattering in the perfect region due to the strong localization in the disordered surface region decreases. This can be mathematically implemented in such a way. For an ordereddisordered separated quantum dot, we can write the effective Hamiltonian for the disordered surface as $H_{S}^{*}=H_{S}-\xi(W)$, while for the inner core perfect region the effective Hamiltonian becomes $H_{C}^{*}=$ $H_{C}-\eta(W)$. Here $\xi(W)=H_{S C}\left(H_{C}-E\right)^{-1} H_{C S}$ and $\eta(W)=H_{C S}\left(H_{S}-E\right)^{-1} H_{S C}$, with $H_{C}$ and $H_{S}$ are the original sub-Hamiltonians for the per-

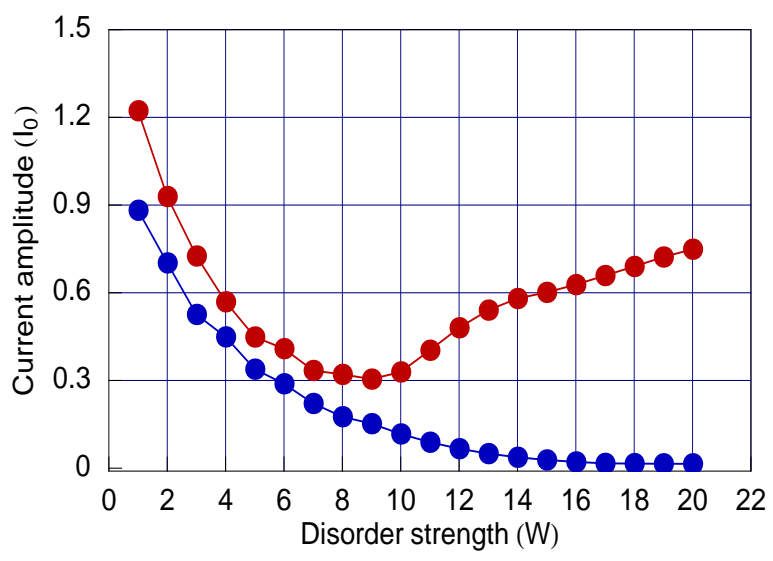

Figure 6: (Color online). Current amplitudes $\left(I_{0}\right)$ as a function of the disorder strength $(W)$ for the multilevel quantum dots with 35 atomic sites in the strong-coupling limit where we take $N_{s}=20$ and $N_{c}=15$. The red and blue curves correspond to the surface and bulk disordered cases, respectively. The typical current amplitudes are computed for $E_{F}=0$.

fect inner core and the disordered surface regions, respectively, and $H_{S C}\left(H_{C S}\right)$ describes their interaction. For $|E|<<W$, we get $\eta \sim H_{C S} H_{S}^{-1} H_{S C}$, leading to $\eta(W) \rightarrow 0$ as $W \rightarrow \infty$. This reveals that, the energy spectrum of an ordered-disordered separated quantum dot with large disorder contains localized tail states with much small and central states with much large values of localization length, contributed approximately by $H_{S}$ and $H_{C}$, respectively. Thus the central states gradually separated from the tail states and delocalized with the increase of the strength of the disorder. Thus we see that, for the coupled ordered-disordered separated quantum dot system, the coupling between the localized states with the inner core extended states is strongly influenced by the strength of the surface disorder, and, this coupling is inversely proportional to the disorder strength $W$. Therefore, in the weak disorder regime the coupling effect is strong, while, the coupling effect becomes less significant in the limit of strong disorder. Accordingly, in the weak disorder regime the electron transport is strongly influenced by the impurities at the surface such that the electron states are scattered more and hence the current amplitude decreases. On the other hand, for the stronger disorder regime the inner core extended states are less influenced by the surface disorder and the coupling effect gradually decreases with the increase of the impurity strength which provide the larger current amplitude in the strong disorder regime. For large enough impurity strength, the inner core extended states are almost unaffected by the impurities at the surface boundary and in that case the current is carried only by these inner core extended states which is the trivial limit. So the exciting limit is the intermediate limit of $W$.

To reveal the finite quantum size effects on the electron transport now we focus our results for the other system size where we consider $N_{s}=24$ and $N_{c}=25$. The results are plotted in Fig. 7, in the limit of strong dot-to-electrode coupling, where all the current amplitudes are computed at the typical bias voltage $V=1.5$ (same as earlier). The red and blue lines denote the identical meaning as in Fig. 6. Both for the surface and bulk disordered systems the current amplitudes show almost the similar behavior for the two different disordered regimes as predicted in Fig. 6. But the significant observation is that the overall current amplitude for this bridge $\left(N_{s}=24\right.$ and $\left.N_{c}=25\right)$ in the case of surface disorder is much larger compared to the results as observed previously i.e., for the surface disordered quantum dot with $N_{s}=20$ and $N_{c}=15$ (see the red line of Fig. (6). This behavior can be explained in such a way. The ratio of the atomic sites in the surface region to the atomic sites in the inner core region for the quantum dot with 49 atomic sites is much smaller than that of the dot with 35 atomic sites and accordingly, the surface effect becomes much less significant for the dot with 49 atomic sites compared to the other dot. Therefore, the current carried by the states in the inner core region for this dot will be less affected by the surface disorder which provides greater current amplitude. Another important observation is that, the typical current amplitude where it goes to a minimum strongly depends on the system size i.e., the number of quantized energy levels or the total number of atomic 
sites in the dot which is clearly visible from the red curves illustrated in Figs. 6 and 7. These results reveal the finite quantum size effects in the study of the electron transport phenomena. The underlying physics behind the location of the minimum in the current versus disorder curve is quite interesting. Two competing mechanisms are there that control the current amplitude. One is the random scattering in the inner core perfect region due to the localization in the disordered surface which tends

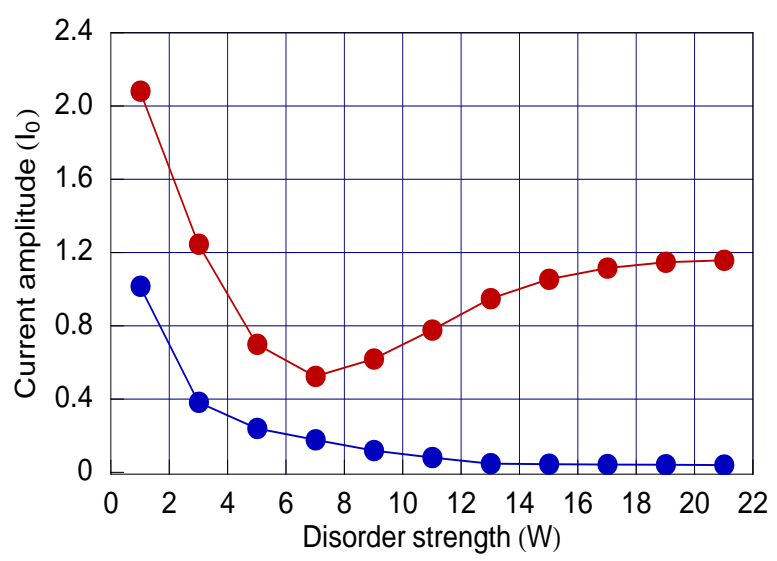

Figure 7: (Color online). Current amplitude $\left(I_{0}\right)$ as a function of the disorder strength $(W)$ for the multilevel quantum dots with 49 atomic sites in the strong-coupling limit where we consider $N_{s}=24$ and $N_{c}=25$. The red and blue curves represent the identical meaning as in Fig. 6. The typical current amplitudes are computed for $E_{F}=0$.

to decrease the current, and, the other one is the vanishing influence of random scattering in the ordered region due to the strong localization in the disordered surface which provides the enhancement of the current. Now, depending on the ratio of the atomic sites in the surface region to the atomic sites in the inner core region, the vanishing effect of random scattering from the ordered states dominates over the non-vanishing effect of random scattering from these states for a particular disorder strength $\left(W=W_{c}\right)$ which provides the location of the minimum in the current versus disorder curve.

A Similar feature of the surface disorder effect is also observed in the limit of weak dot-to-electrode coupling strength with reduced current amplitudes for these quantum dots and in the obvious reason here we do not describe these results once again. Throughout our study of the surface disorder effect on the electron transport we compute all the typical current amplitudes for the equilibrium Fermi energy
$E_{F}=0$ only, and, this peculiar behavior will also be observed for the other values of $E_{F}$.

\section{Concluding remarks}

In conclusion of this article, we have introduced a parametric approach based on the tight-binding model to study the electron transport characteristics through some multilevel quantum dots. From our results we can predict that the electron transport is significantly influenced by (a) the number of quantized energy levels in the dots, (b) the location of the equilibrium Fermi energy $E_{F}$, (c) the dot-to-electrodes coupling strength and (d) the surface disorder. All the results have been performed by using the Green's function technique and this technique can be used to study the electron transport in any complicated system, like complicated organic molecule, quantum wire, array of quantum dots etc., which bridges the two reservoirs.

The conductance shows sharp resonant peaks for the weak-coupling limit (red curves of Fig. 2), while, they get broadened in the limit of strong-coupling (blue curves of Fig. 2). Such increment of the resonant widths is due to the broadening of the quantized energy levels of the dots, where the contribution comes from the imaginary parts of the two self energies $\Sigma_{S}$ and $\Sigma_{D}$ [42].

In the determination of the current, we have seen that the current shows staircase-like structures with sharp steps (red lines in Fig. 3) in the limit of weakcoupling, while it (current) varies quite continuously (red lines in Fig. 4) and achieves very large value in the strong-coupling limit.

Next in the description of the noise power of the current fluctuations we have noticed that whether the shot noise lies in the Poisson regime $(F=1)$ or in the sub-Poisson regime $(F<1)$ strongly depends on the location of the Fermi energy $E_{F}$ and the dotto-electrodes coupling strength.

Finally, in the study of the surface disorder effect we have explored a novel transport phenomenon in which the current amplitude increases with the increase of the surface disorder strength in the strong disorder regime, while, the current amplitudes decreases in the weak disorder regime. Such an anomalous behavior has not been pointed out previously in the literature where the transport properties have been described through the bridge systems. This feature is completely opposite to that of the bulk disordered system in which the current amplitude decays gradually with the increase of the impurity strength and eventually drops to zero. 
Several realistic assumptions have been made in the present study. More studies are expected to take into account the Schottky effect which comes from the charge transfer across the dot-electrode interfaces, the static Stark effect, which is considered for the modification of the electronic structure of the bridging system due to the applied bias voltage (essential especially for higher voltages). However all these effects can be included into our framework by a simple generalization of the presented formalism. In this article we have also neglected the effects of all the inelastic scattering processes and the Coulomb correlation to characterize the electron transport through such quantum dots.

\section{References}

[1] A. T. Tilke, F. C. Simmel, H. Lorenz, R. H. Blick, and J. P. Kotthaus, Phys. Rev. B 68, 075311 (2003).

[2] A. W. Holleitner, C. R. Decker, H. Qin, K. Eberl, and R. H. Blick, Phys. Rev. Lett. 87, 256802 (2001).

[3] A. W. Holleitner, R. H. Blick, A. K. Huttel, K. Eber, and J. P. Kotthaus, Science 297, 70 (2002).

[4] W. Z. Shangguan, T. C. Au Yeung, Y. B. $\mathrm{Yu}$, and C. H. Kam, Phys. Rev. B 63, 235323 (2001).

[5] A. I. Yanson, G. Rubio-Bollinger, H. E. van den Brom, N. Agrait, and J. M. van Ruitenbeek, Nature (London) 395, 780 (1998).

[6] A. Aviram and M. Ratner, Chem. Phys. Lett. 29, 277 (1974).

[7] T. Dadosh, Y. Gordin, R. Krahne, I. Khivrich, D. Mahalu, V. Frydman, J. Sperling, A. Yacoby, and I. Bar-Joseph, Nature 436, 677 (2005).

[8] R. M. Metzger et al., J. Am. Chem. Soc. 119, 10455 (1997).

[9] C. M. Fischer, M. Burghard, S. Roth, and K. V. Klitzing, Appl. Phys. Lett. 66, 3331 (1995).

[10] J. Chen, M. A. Reed, A. M. Rawlett, and J. M. Tour, Science 286, 1550 (1999).

[11] M. A. Reed, C. Zhou, C. J. Muller, T. P. Burgin, and J. M. Tour, Science 278, 252 (1997).
[12] M. Magoga and C. Joachim, Phys. Rev. B 59, 16011 (1999).

[13] J.-P. Launay and C. D. Coudret, in: A. Aviram and M. A. Ratner (Eds.), Molecular Electronics, New York Academy of Sciences, New York, (1998).

[14] R. Baer and D. Neuhauser, Chem. Phys. 281, 353 (2002).

[15] R. Baer and D. Neuhauser, J. Am. Chem. Soc. 124, 4200 (2002).

[16] R. H. Goldsmith, M. R. Wasielewski, and M. A. Ratner, J. Phys. Chem. B 110, 20258 (2006).

[17] M. Ernzerhof, H. Bahmann, F. Goyer, M. Zhuang, and P. Rocheleau, J. Chem. Theory Comput. 2, 1291 (2006).

[18] Y. M. Blanter and M. Büttiker, Phys. Rep. 336, 1 (2000).

[19] S. N. Yaliraki, A. E. Roitberg, C. Gonzalez, V. Mujica, and M. A. Ratner, J. Chem. Phys. 111, 6997 (1999).

[20] M. Di Ventra, S. T. Pantelides, and N. D. Lang, Phys. Rev. Lett. 84, 979 (2000).

[21] Y. Xue, S. Datta, and M. A. Ratner, J. Chem. Phys. 115, 4292 (2001).

[22] J. Taylor, H. Guo, and J. Wang, Phys. Rev. B 63, 245407 (2001).

[23] P. A. Derosa and J. M. Seminario, J. Phys. Chem. B 105, 471 (2001).

[24] P. S. Damle, A. W. Ghosh, and S. Datta, Phys. Rev. B 64, R201403 (2001).

[25] (a) M. Elstner et al., Phys. Rev. B 58, 7260 (1998). (b) T. Frauenheim et al., J. Phys.: Condens. Matter 14, 3015 (2002).

[26] (a) P. Hohenberg and W. Kohn, Phys. Rev. 136, B864 (1964). (b) W. Kohn and L. J. Sham, Phys. Rev. 140, A1133 (1965).

[27] N. Sai, M. Zwolak, G. Vignale, and M. D. Ventra, Phys. Rev. Lett. 94, 186810 (2005).

[28] N. Bushong, N. Sai, and M. D. Ventra, Nano Lett. 5, 2569 (2005).

[29] M. D. Ventra and T. N. Todorov, J. Phys.: Condens. Matter 16, 8025 (2004). 
[30] V. Mujica, M. Kemp, and M. A. Ratner, J. Chem. Phys. 101, 6849 (1994).

[31] V. Mujica, M. Kemp, A. E. Roitberg, and M. A. Ratner, J. Chem. Phys. 104, 7296 (1996).

[32] S. K. Maiti, Solid State Phenomena 155, 71 (2009).

[33] M. P. Samanta, W. Tian, S. Datta, J. I. Henderson, and C. P. Kubiak, Phys. Rev. B 53, R7626 (1996).

[34] M. Hjort and S. Staftröm, Phys. Rev. B 62, 5245 (2000).

[35] K. Walczak, Cent. Eur. J. Chem. 2, 524 (2004).

[36] K. Walczak, Phys. Stat. Sol. (b) 241, 2555 (2004).

[37] C. Y. Yang, J. W. Ding, and N. Xu, Physica B 394, 69 (2007).

[38] H. B. Chen and J. W. Ding, Physica B 403, 2015 (2008).

[39] L. P. Kouwenhoven, C. M. Marcus, P. L. McEuen, S. Tarucha, R. M. Westervelt, and N. S. Wingreen, in Mesoscopic Electron Transport: Proc. NATO Advanced Study Institutes (NATO Advanced Study Institute, Series E: Applied Sciences) 345, (1997).

[40] J. X. Zhong and G. M. Stocks, Nano. Lett. 6, 128 (2006).

[41] J. X. Zhong and G. M. Stocks, Phys. Rev. B 75, 033410 (2007).

[42] S. Datta, Electronic transport in mesoscopic systems, Cambridge University Press, Cambridge (1997).

[43] W. Tian, S. Datta, S. Hong, R. Reifenberger, J. I. Henderson, and C. I. Kubiak, J. Chem. Phys. 109, 2874 (1998). 